\title{
Development and validation of a self-administered neck mobility assessment tool (S-ROM-Neck) in chronic neck pain patients
}

Citation for published version (APA):

Langenfeld, A., Bastiaenen, C., Sieben, J., \& Swanenburg, J. (2018). Development and validation of a self-administered neck mobility assessment tool (S-ROM-Neck) in chronic neck pain patients. Musculoskeletal science and practice, 37, 75-79. https://doi.org/10.1016/j.msksp.2018.04.004

Document status and date:

Published: 01/10/2018

DOI:

10.1016/j.msksp.2018.04.004

Document Version:

Publisher's PDF, also known as Version of record

Document license:

Taverne

Please check the document version of this publication:

- A submitted manuscript is the version of the article upon submission and before peer-review. There can be important differences between the submitted version and the official published version of record.

People interested in the research are advised to contact the author for the final version of the publication, or visit the DOI to the publisher's website.

- The final author version and the galley proof are versions of the publication after peer review.

- The final published version features the final layout of the paper including the volume, issue and page numbers.

Link to publication

\footnotetext{
General rights rights.

- You may freely distribute the URL identifying the publication in the public portal. please follow below link for the End User Agreement:

www.umlib.nl/taverne-license

Take down policy

If you believe that this document breaches copyright please contact us at:

repository@maastrichtuniversity.nl

providing details and we will investigate your claim.
}

Copyright and moral rights for the publications made accessible in the public portal are retained by the authors and/or other copyright owners and it is a condition of accessing publications that users recognise and abide by the legal requirements associated with these

- Users may download and print one copy of any publication from the public portal for the purpose of private study or research.

- You may not further distribute the material or use it for any profit-making activity or commercial gain

If the publication is distributed under the terms of Article 25fa of the Dutch Copyright Act, indicated by the "Taverne" license above, 
Technical and measurement report

\title{
Development and validation of a self-administered neck mobility assessment tool (S-ROM-Neck) in chronic neck pain patients
}

\author{
Anke Langenfeld $^{\mathrm{a}, \mathrm{b}, *}$, Caroline Bastiaenen ${ }^{\mathrm{a}, \mathrm{c}}$, Judith Sieben ${ }^{\mathrm{a}, \mathrm{d}}$, Jaap Swanenburg ${ }^{\mathrm{b}, \mathrm{e}}$ \\ ${ }^{\text {a } C A P H R I ~ S c h o o l ~ f o r ~ P u b l i c ~ H e a l t h ~ a n d ~ P r i m a r y ~ C a r e, ~ M a a s t r i c h t ~ U n i v e r s i t y, ~ M a a s t r i c h t, ~ T h e ~ N e t h e r l a n d s ~}$ \\ b Interdisciplinary Spinal Research ISR, Department of Chiropractic Medicine, Balgrist University Hospital, Zürich, CH, Switzerland \\ c Department of Epidemiology, Maastricht University, Maastricht, The Netherlands \\ d Department of Anatomy \& Embryology, Maastricht University, Maastricht, The Netherlands \\ e Physiotherapy Occupational Therapy Research Centre, Directorate of Research and Education, University Hospital Zurich, Zurich, Switzerland
}

\section{A R T I C L E I N F O}

\section{Keywords:}

Patient's perspective

Range of motion

Cervical spine

Validity

\begin{abstract}
A B S T R A C T
Background: Cervical spine (CS) range of motion (ROM) is commonly used to assess neck pain. However, this measurement is often limited by the clinician's experience and perception. Therefore, the integration of perceptual feedback of the patient can optimize and personalize treatment.

Objective: Develop and validate a questionnaire (S-ROM-Neck) to evaluate ROM of the CS from the patient's perspective.

Design: Validation study.

Methods: The assessment tool was developed and optimized during pretest sessions. Reliability and construct validity of the questionnaire were tested. 50 participants (age $>18$ years) with neck pain for $>90$ days, able to fluently speak, read, and write in German were included. Exclusion criteria included any condition that limited manual therapy to the CS. Participants completed S-ROM-Neck twice within seven days, along with the visual analogue scale (VAS) for pain intensity and the German version of the Neck Disability Index (NDI-G). The relative reliability, internal consistency, and absolute reliability were analyzed, and Bland-Altman plots were generated. Construct validity was established by correlating the total score of S-ROM-Neck with VAS and NDI-G based on pre-set hypotheses.

Results: S-ROM-Neck demonstrated moderate reliability with an intraclass correlation coefficient of 0.718 (Cronbach's alpha of 0.83 ). There was a medium negative correlation between VAS and S-ROM-Neck [Spearman's rank correlation coefficient $\left(r_{s}\right)=-0.30, p=0.031$ ] and the NDI-G and S-ROM-Neck $\left(r_{s}=-0.40\right.$, $p=0.002$ ).

Conclusions: These results indicate the reliability and internal consistency of S-ROM-Neck. This scale provides patient feedback and perspectives to optimize assessment of neck pain.
\end{abstract}

\section{Introduction}

Neck pain is a common cause of disability that is associated with reduced range of motion (ROM) of the cervical spine (CS) (Hoy et al., 2014; Rudolfsson et al., 2012). CS ROM is a common tool to assess the degree of neck pain and the level of impairment as well as to monitor progress and evaluate the effectiveness of treatment and interventions (Fletcher and Bandy, 2008; de Koning et al., 2008).

Typically, CS ROM assessment is conducted and interpreted by a trained clinician. However, patient perception provides an additional understanding of the needs and problems of the patients by translating clinical data into a patient-centered measurement (Wiklund, 2004).
Furthermore, the patient's point of view can add valuable information to an examination by building a holistic picture of the patient's problems, possible needs, and expectations (Bee et al., 2016; Bishop et al., 2013; Scherer et al., 2010).

Self-reported ROM tools have been effectively utilized to evaluate other body parts (Beirer et al., 2015; Collins et al., 2014; Gioe et al., 2009; Khanna et al., 2011; Saund et al., 2012; Uribe et al., 2016). Furthermore, a previous study employed a similar approach to that used in the present study; however, the prior approach focused on pain intensity induced by head movement (Lauche et al., 2014).

Until now, no study has systematically assessed the ability of patients to rate their own perception of CS mobility. Such a tool would

\footnotetext{
* Corresponding author. Balgrist University Clinic, Forchstrasse 340, 8008, Zurich, Switzerland.

E-mail address: ankelangenfeld@balgrist.ch (A. Langenfeld).
} 
allow the addition of perceptual feedback and patient-specific information on movement restrictions of the CS, thereby improving the understanding of patient limitations, facilitating the selection of therapeutic options by clinicians, and optimizing cooperation between clinicians and patients.

This study aimed to develop a self-reported measurement tool to assess ROM of the CS.

\section{Methods}

\subsection{Development and pretesting}

The self-reported CS ROM questionnaire (S-ROM-Neck) was developed to evaluate CS ROM because patient assessment in daily practice often lacks assessment of CS mobility from the patient's point of view. Normally, during the first appointment with a patient with neck pain, the clinician assesses CS mobility and the patient is asked to describe pain and movement restrictions. However, to the best of our knowledge, no current questionnaire systematically assesses patient responses. The scale incorporates six questions targeting primary movements of the CS (i.e., flexion, extension, rotation, and lateral flexion). Patients were asked to actively perform each neck movement and then rate the restriction they perceived during the movement. This was done for each of the 6 movement directions. Perceived restriction could have been due to pain, stiffness and or tension. The questions were developed by clinicians and later tested and modified, if needed, during a pretest.

The tool was based upon a visual analogue scale (VAS) with two anchors. The left side contained the words "not at all" to indicate severe ROM restriction and the right side contained the words "as far as I want to" to indicate maximum ROM. Explanatory pictures accompany each question. The total score is calculated by adding the individual scores (minimum score $[600]=$ no restrictions; maximum score $[0]=$ total restriction).

Ten participants who fulfilled the same inclusion and exclusion criteria as the criteria for the main study were recruited for a pretest session to provide feedback on usability components. Based on the pretest feedback, two of the explanatory pictures were changed. These images accompanied the question "Side bending left" and "Side bending right." The person in the pictures was shown from the front. However, the participants reported confusion because the arrow pointed in the opposite direction as the patients were asked to move their heads. Therefore, the images were flipped to indicate the accurate movement (Additional File 1).

\subsection{Participants and procedures}

The study protocol was approved by the Ethics Committee of the "X". Eligible patients were asked about their interest in participating in the study. Those who wished to participate in this study received information and signed an informed consent form prior to study participation. Eligibility criteria were age $>18$ years, neck pain lasting $>90$ days, and ability to fluently speak, read, and write in German. Participants with any contraindications or limitations to manual therapy to the CS, such as multi-level nerve root pathologies, recent trauma to the CS, spinal cord damage, and active cancer, were excluded from the study (Rushton et al., 2012).

\subsection{Measures}

Demographic data of weight, sex, age, height, and duration of complaints were collected at baseline using a form that was completed by each participant. The VAS and the German version of the Neck Disability Index (NDI-G) were administered by a licensed physiotherapist. Participants filled out the forms by themselves. S-ROMNeck assessment was repeated after 3-7 days. Participants received a prepaid envelope at the initial appointment and were instructed to return the questionnaire upon completion. If the questionnaire was not returned after four days, the participants received a reminder telephone call.

\subsection{VAS}

VAS is a valid and reliable outcome measure to assess pain intensity (Price et al., 1983). The left side of the 100-mm scale is illustrative of "no pain" and the far right side is "extreme pain" (Williamson and Hoggart, 2005).

\subsection{NDI-G}

NDI-G is a valid and reliable questionnaire to assess self-rated disability in patients experiencing neck pain (Swanenburg et al., 2014). The scores range from 0 to 50, with a lower score associated with low self-rated disability (Vernon and Mior, 1991; (Swanenburg et al., 2014).

Hypothesis. There is no gold standard for the measurement of patient perception on the self-reported ROM; hence, the following two hypotheses were created to assess the validity of the tool:

1. There is a negative correlation between S-ROM-Neck and NDI $(-0.3$ to -0.5 )

2. There is a negative correlation between S-ROM-Neck and VAS $(-0.3$ to -0.5$)$

These hypotheses were based on the assumption that decreases in pain and functional disability would result in an increase in CS mobility.

\subsection{Data analysis}

Data were stored and analyzed using the IBM SPSS 22 statistical software package (SPSS, Inc., Chicago, IL, USA). Descriptive statistics were used to describe the baseline characteristics of the participants. The reliability of S-ROM-Neck was assessed using an intraclass correlation coefficient $\left(\mathrm{ICC}_{\text {agreement }}\right.$ ) with $95 \%$ confidence intervals (CI) (Darter et al., 2013; Koo and Li, 2016). Cronbach's alpha was used to evaluate internal consistency (Bland and Altman, 1997). The standard error of measurement (SEM) and smallest detectable change (SDC) were used to determine the absolute reliability of S-ROM-Neck (Bruton et al., 2000; Darter et al., 2013). Bland-Altman plots were generated to assess agreement of the repeated S-ROM-Neck measures (Bland and Altman, 1986). To establish construct validity, the total score of S-ROM-Neck was correlated with VAS and NDI-G based on pre-set hypotheses (de Vet et al., 2011). The floor and ceiling effects of S-ROM-Neck were used to assess content validity.

\section{Results}

A total of 50 participants (mean age, $43.62 \pm 14.94$ years; mean height, $169.94 \pm 10.09 \mathrm{~cm}$; mean weight, $69.78 \pm 17.57 \mathrm{~kg}$; $35 \mathrm{fe}-$ male participants) with a duration of neck pain of $>90$ days were included in this study. The mean overall S-ROM-Neck score was $42.43 \pm 10.70 \mathrm{~mm}$, the mean overall VAS score was $35.7 \pm 22.8 \mathrm{~mm}$, and the mean NDI score was $13.16 \pm 5.92$. Two patients had one missing item, which was not replaced.

S-ROM-Neck demonstrated reliability with an ICC value of 0.718 (95\% CI $=0.47-0.88)$ and Cronbach's alpha of 0.83 . The SEM was $3.1 \mathrm{~mm}$, and the SDC was $8.7 \mathrm{~mm}$. The Bland-Altman plot indicated that all points, except two, were located within $95 \%$ limits of agreement for test-retest, and visual inspection showed no tendency toward heteroscedasticity (see Fig. 1).

Spearman's rho between VAS and S-ROM-Neck showed a moderate negative correlation $\left(r_{s}=-0.31, p=0.026\right)$, similar to NDI and $S$ - 


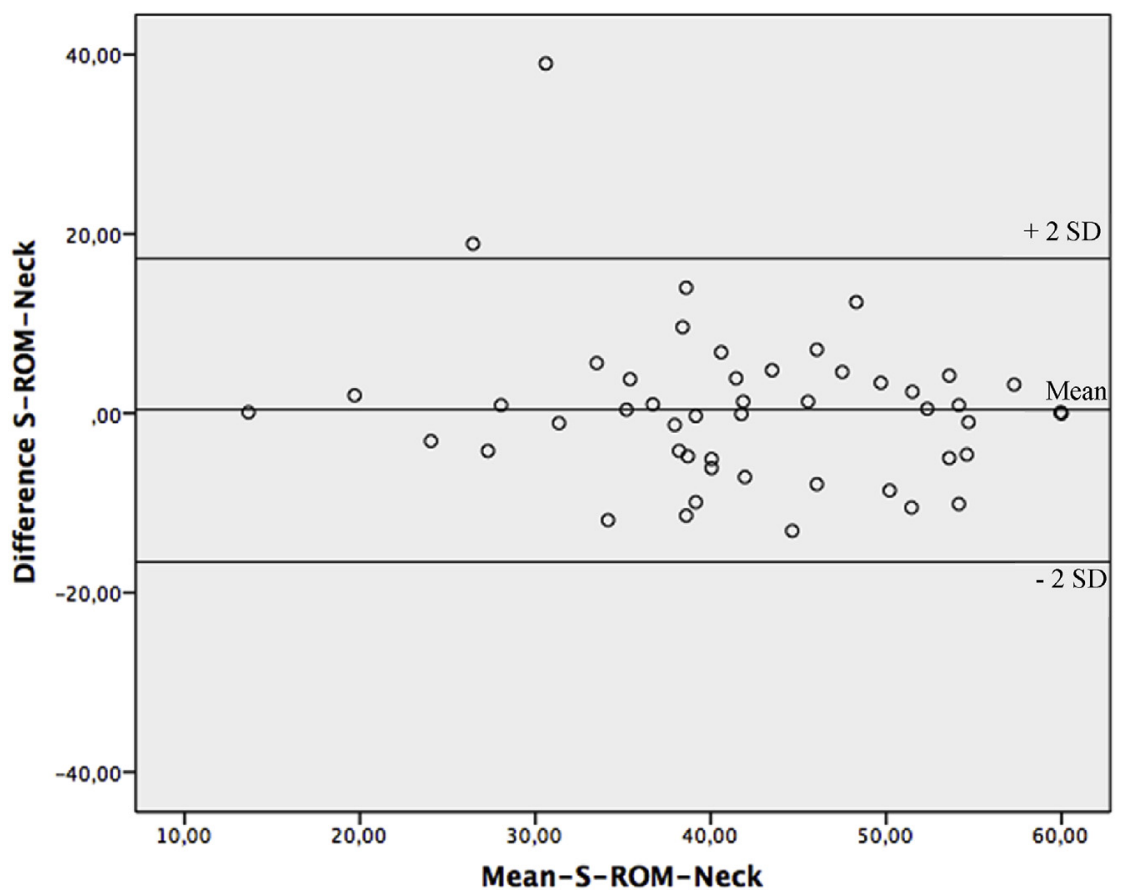

Fig. 1. Bland-Altman plot of S-ROM-Neck.

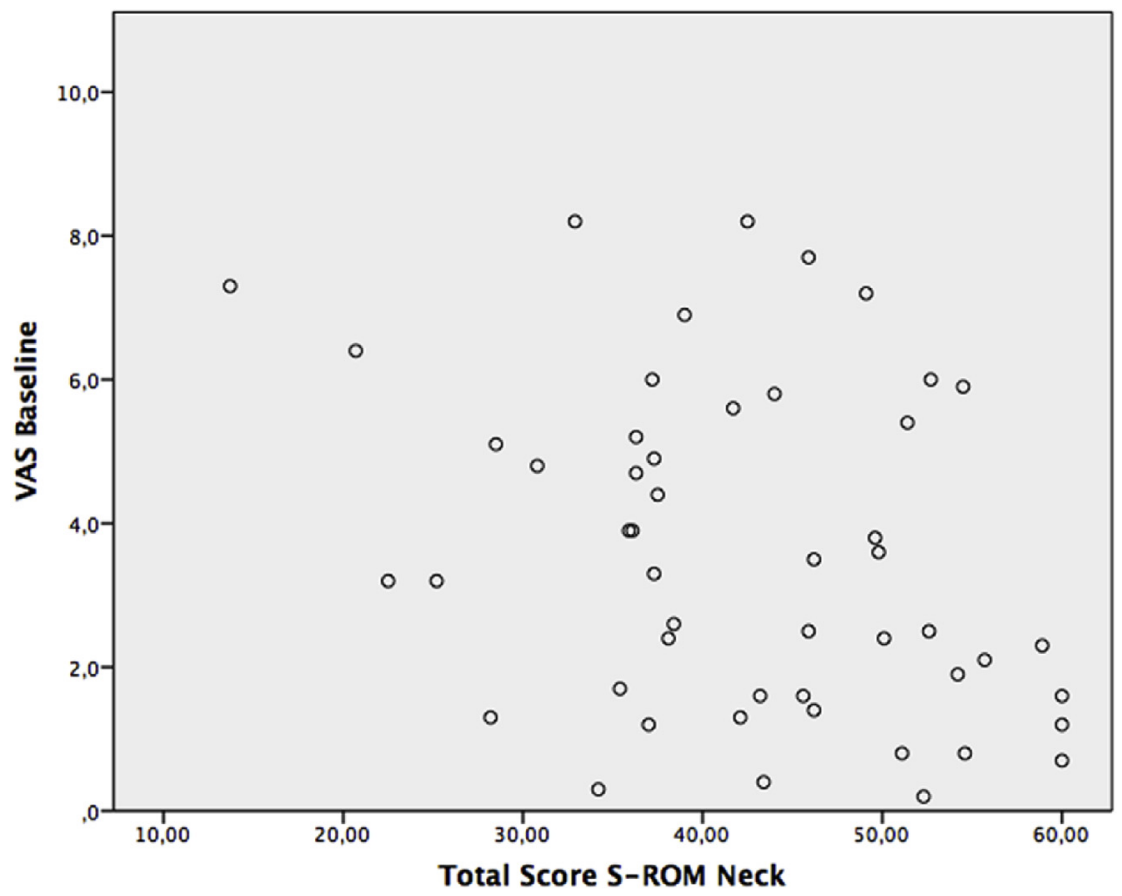

Fig. 2. Scatterplot of VAS vs. total score of S-ROM-Neck.

ROM-Neck $\left(r_{s}=-0.42, p=0.002\right)$ (see Figs. 2 and 3). There were no floor or ceiling effects of S-ROM-Neck.

\section{Discussion}

We aimed to develop a patient-reported assessment tool (S-ROMNeck) to measure CS ROM from the patient's perspective. This self-assessment adds unique, patient-specific information to support clinical assessment and evaluation. Furthermore, the reliability and construct validity of the assessment tool were evaluated on the basis of the study hypotheses. The Hypothesis that there is a negative correlation between
S-ROM-Neck and both external anchors (NDI and VAS) was confirmed. Assessment of the reliability of the novel S-ROM-Neck tool was investigated with an ICC of 0.718 and Cronbach's alpha of 0.83 (Bland and Altman, 1997; Terwee et al., 2007).

A literature review revealed four studies that compared the ratings of ROM of different body parts by the clinicians and patients (Collins et al., 2014; Gioe et al., 2009; Khanna et al., 2011; Uribe et al., 2016), but only two of these studies developed and evaluated novel measures for validity and reliability (elbow disorders and mouth-opening) (Beirer et al., 2015; Saund et al., 2012). For the total score and subscales, Beirer et al. (2015) calculated the ICC, which ranged from 0.76 to 0.81 , and 


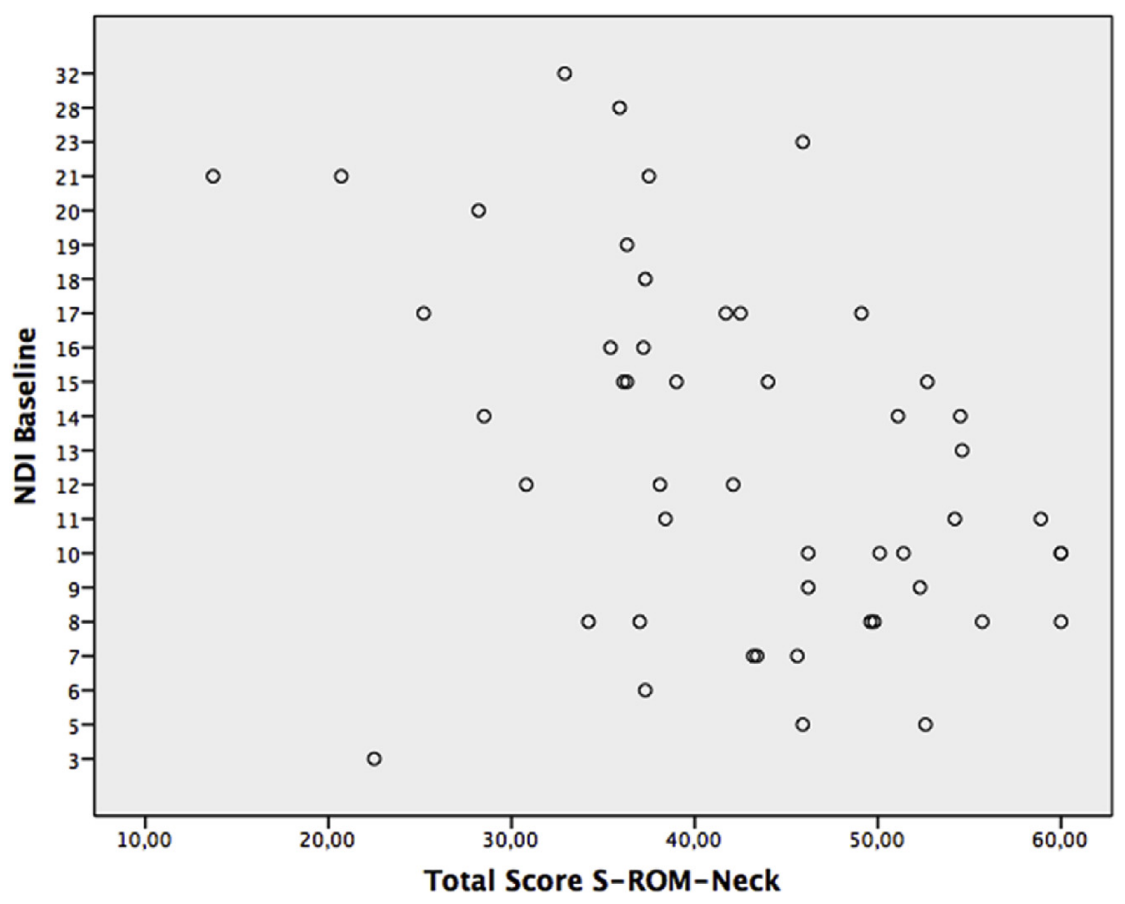

Fig. 3. Scatterplot of baseline NDI-G vs. total score of S-ROM-Neck.

Cronbach's alpha, which ranged from 0.83 to 0.92 . They found a correlation between the Elbow Self-Assessment Score and Oxford Elbow Score that ranged from -0.80 to -0.84 . Saund et al. (2012) reported an ICC of $0.92(95 \% \mathrm{CI}=0.88-0.95$; Pearson's correlation coefficient $=0.86-0.90$ ). The instrument proposed in the present study achieved an ICC of 0.71. Although this value is lower than that reported by Beirer and Saund, it was sufficient to consider the instrument as moderately reliable (Beirer et al., 2015). The correlations were medium, indicating that S-ROM-Neck provides additional information that was not assessed by VAS or NDI. This additional information may offer valuable insight into patient-specific care and treatment.

The decision to assess construct validity rather than criterion validity was intentional. Criterion validity is used if there is a gold standard to compare the results of a newly developed tool (Mokkink et al., 2010). On the contrary, there is no such gold standard to assess construct validity (Mokkink et al., 2010). Subsequently, the results of the analysis were compared with a pre-set Hypothesis (Mokkink et al., 2010). The construct that we aimed to measure was the patient's perspective on CS ROM. Although there is a reliable standard for CS ROM measurement (Fletcher and Bandy, 2008; de Koning et al., 2008), there is currently no gold standard to measure CS ROM from the patient's perspective.

Self-reported ROM tools have been effectively utilized in shoulder, elbow, jaw, and knee assessment, (Beirer et al., 2015; Collins et al., 2014; Gioe et al., 2009; Khanna et al., 2011; Saund et al., 2012; Uribe et al., 2016), but have used various methods of assessment thus direct comparison is difficult. Interestingly, Lauche et al., 2014 study was most similar to our study, but patients only assessed pain during the movement and pain may not necessarily indicate restriction of movement. Nevertheless, results ie (mean values/600 mm) in the current study were very similar to POM in Lauche's study 2014. This may have been because pain could have been one of the causes of the restriction rating in the S-ROM Neck. Since movement restrictions can be caused by other factors than pain, the S-ROM-Neck may provide additional information and future research should investigate this.

A difficulty associated with CS mobility is the combination of movements that naturally occur in the spine (e.g., a combination of lateral flexion and rotation) (Ishii et al., 2006), which may cause difficulty in using a single plane to judge movement of the CS. Intentionally, participants were not instructed on how to conduct the movement, so that they would execute the movement organically to reflect daily movement. Therefore, movements were supported only with pictures rather than with instructions or verbal cues. Participants were not asked to conduct warm-up movements nor were any restrictions placed on how often they executed the movement.

This study had a minimum sample size of 50 patients with chronic neck pain (de Vet et al., 2011). ICC was moderate, which might be due to the homogenous sample. Therefore, future studies are required for assessing psychometric properties and performing factor analysis in a larger and more heterogeneous group of patients. Nevertheless, the ICC of 0.718 is a positive rating of reliability in a sample of 50 participants (Terwee et al., 2007). A study focusing on pain intensity induced by head movement used analogue proceedings (Lauche et al., 2014). Since movement restrictions can also be caused by other factors than pain, it is difficult to compare it with the S-ROM-Neck. Moreover having neck pain does not mean that the patient experiences movement restrictions of the cervical spine. Future research should determine differences between those questionnaires.

Future studies could combine both questionnaires and evaluate each in a more heterogeneous sample of neck pain patients. Furthermore, because we did not combine the S-ROM-Neck measurements to objective measurements (e.g., ROM), we recommend to do so in future studies in order to assess S-ROM-Neck to acquire additional information.

\section{Conclusion}

Our findings support the reliability and internal consistency of the S-ROM-Neck instrument. Negative correlations of VAS and NDI with the S-ROM-Neck indicate that a larger CS ROM is associated with less pain and disability. Most importantly, the S-ROM-Neck tool is a useful measure that allows the input of patient-specific information to support a more comprehensive assessment of patients with neck pain.

\section{Funding}

The authors received no specific grant from any funding agency in 
the public, commercial, or not-for-profit sectors in regard to this report.

\section{Conflicts of interest}

None declared.

\section{Statement of institutional review board approval of the study protocol}

The study was approved by the ethics commission of the Canton of Zurich (PB_2016-00164) and registered at clinicaltrials.gov (NCT02754934).

\section{References}

Bee, P., McBeth, J., MacFarlane, G.J., Lovell, K., 2016. Managing chronic widespread pain in primary care: a qualitative study of patient perspectives and implications for treatment delivery. BMC Musculoskelet. Disord. 17, 354. http://dx.doi.org/10.1186/ s12891-016-1194-5.

Beirer, M., Friese, H., Lenich, A., Cr??nlein, M., Sandmann, G.H., Biberthaler, P., et al. 2015. The Elbow Self-Assessment Score (ESAS): development and validation of a new patient-reported outcome measurement tool for elbow disorders. Knee Surg. Sport Traumatol. Arthrosc. http://dx.doi.org/10.1007/s00167-015-3647-z.

Bishop, M.D., Mintken, P.E., Bialosky, J.E., Cleland, J a, 2013. Patient expectations of benefit from interventions for neck pain and resulting influence on outcomes. J. Orthop. Sports Phys. Ther. 43, 457-465. http://dx.doi.org/10.2519/jospt.2013. 4492.

Bland, J., Altman, D., 1986. Statistical methods for assessing agreement between two methods of clinical measurement. Lancet 327, 307-310. http://dx.doi.org/10.1016/ S0140-6736(86)90837-8.

Bland, J.M., Altman, D.G., 1997. Cronbach's alpha. BMJ 314, 572. http://dx.doi.org/10. 1136/bmj.314.7080.572.

Bruton, A., Conway, J.H., Holgate, S.T., 2000. Reliability: what is it, and how is it measured? Physiotherapy 86, 94-99. http://dx.doi.org/10.1016/S0031-9406(05) 61211-4.

Collins, J.E., Rome, B.N., Daigle, M.E., Lerner, V., Katz, J.N., Losina, E., 2014. A comparison of patient-reported and measured range of motion in a Cohort of total knee replacement Patie. J. Arthroplast 29, 1378-1382. http://dx.doi.org/10.1016/j.arth. 2014.02.023.A.

Darter, B.J., Rodriguez, K.M., Wilken, J.M., 2013. Test-retest reliability and minimum detectable change using the K4b2: oxygen consumption, gait efficiency, and heart rate for healthy adults during submaximal walking. Res. Q. Exerc Sport 84, 223-231. http://dx.doi.org/10.1080/02701367.2013.784720.

Fletcher, J.P., Bandy, W.D., 2008. Intrarater reliability of CROM measurement of cervical spine active range of motion in persons with and without neck pain. J. Orthop. Sports Phys. Ther. 38, 640-645. http://dx.doi.org/10.2519/jospt.2008.2680.

Gioe, T.J., Pomeroy, D., Suthers, K., Singh, J.A., 2009. Can patients help with long-term total knee arthroplasty surveillance? Comparison of the American Knee Society Score self-report and surgeon assessment. Rheumatology 48, 160-164. http://dx.doi.org/ 10.1093/rheumatology/ken439.

Hoy, D., March, L., Woolf, A., Blyth, F., Brooks, P., Smith, E., et al., 2014. The global burden of neck pain: estimates from the global burden of disease 2010 study. Ann. Rheum. Dis. 73, 1309-1315. http://dx.doi.org/10.1136/annrheumdis-2013-204431.
Ishii, T., Mukai, Y., Hosono, N., Sakaura, H., Fujii, R., Nakajima, Y., et al., 2006. Kinematics of the cervical spine in lateral bending: in vivo three-dimensional analysis. Spine 31, 155-160. http://dx.doi.org/10.1097/01.brs.0000195173.47334.1f (Phila Pa 1976).

Khanna, G., Singh, J.A., Pomeroy, D.L., Gioe, T.J., 2011. Coparison of patient-reported and clinician-assessed outcomes following total knee arthroplasty. J. Bone Jt. Surg. $117,1-7$.

de Koning, C.H.P., van den Heuvel, S.P., Staal, J.B., Smits-Engelsman, B.C.M., Hendriks, E.J.M., 2008. Clinimetric evaluation of active range of motion measures in patients with non-specific neck pain: a systematic review. Eur. Spine J. 17, 905-921. http:// dx.doi.org/10.1007/s00586-008-0656-3.

Koo, T.K., Li, M.Y., 2016. A guideline of selecting and reporting intraclass correlation coefficients for reliability research. J. Chiropr. Med. 15, 155-163. http://dx.doi.org/ 10.1016/j.jcm.2016.02.012.

Lauche, R., Cramer, H., Langhorst, J., Michalsen, A., Dobos, G.J., 2014. Reliability and validity of the pain on movement questionnaire (POM) in chronic neck pain. Pain Med. (United States) 15, 1850-1856. http://dx.doi.org/10.1111/pme.12534.

Mokkink, L.B., Terwee, C.B., Patrick, D.L., Alonso, J., Stratford, P.W., Knol, D.L., et al., 2010. The COSMIN study reached international consensus on taxonomy, terminology, and definitions of measurement properties for health-related patient-reported outcomes. J. Clin. Epidemiol. 63, 737-745. http://dx.doi.org/10.1016/j.jclinepi. 2010.02.006.

Price, D., McGrath, P., Rafii, A., Buckingham, B., 1983. The validation of visual analogue scales as ratio scale measures for chronic and experimental pain. Pain 17, 45-56.

Rushton, A., Rivett, D., Carlesso, L., Flynn, T., Hing, W., Kerry, R., 2012. Internationa Framework for the Examination of the Cervical Region for Potential Cervical Arterial Dysfunction Prior to Orthopeadic Manual Therapy Intervention. pp. 1-37. http:// www.ifompt.com/site/ifompt/IFOMPT Examination cervical spine doc September 2012 definitive.pdf, Accessed date: 6 February 2015.

Rudolfsson, T., Björklund, M., Djupsjöbacka, M., 2012 Feb. Range of motion in the upper and lower cervical spine in people with chronic neck pain. Man. Ther. 17 (1), 53-59. http://dx.doi.org/10.1016/j.math.2011.08.007. Epub 2011 Sep 25.

Saund, S.S.D., Pearson, D., Dietrich, T., 2012. Reliability and validity of self-assessment of mouth opening: a validation study. BMC Oral Health 12, 1. http://dx.doi.org/10. 1186/1472-6831-12-48.

Scherer, M., Schaefer, H., Blozik, E., Chenot, J.-F., Himmel, W., 2010. The experience and management of neck pain in general practice: the patients' perspective. Eur. Spine J. 19, 963-971. http://dx.doi.org/10.1007/s00586-010-1297-x.

Swanenburg, J., Humphreys, K., Langenfeld, A., Brunner, F., Wirth, B., 2014. Validity and reliability of a German version of the neck disability Index (NDI-G). Man. Ther. 19, 52-58. http://dx.doi.org/10.1016/j.math.2013.07.004.

Terwee, C.B., Bot, S.D.M., de Boer, M.R., van der Windt, D a, WM, Knol, D.L., Dekker, J., et al., 2007. Quality criteria were proposed for measurement properties of health status questionnaires. J. Clin. Epidemiol. 60, 34-42. http://dx.doi.org/10.1016/j. jclinepi.2006.03.012.

Uribe, B., El, Bitar Y., Wolf, B.R., Bollier, M., Kuhn, J.E., Hettrich, C.M., 2016. Agreement between patient self-assessment and physician assessment of shoulder range of motion. J. Shoulder Elb. Surg. http://dx.doi.org/10.1016/j.jse.2016.02.010.

Vernon, H., Mior, S., 1991. The neck disability Index: a study of reliability and validity. J. Manip. Physiol. Ther. 14, 409-415.

de Vet, H.C.W., Terwee, C.B., Mokkink, L.B., Knol, D.L., 2011. Measurements in Medicine Cambridge University Press.

Wiklund, I., 2004. Assessment of patient-reported outcomes in clinical trials: the example of health-related quality of life. Fundam. Clin. Pharmacol. 18, 351-363. http://dx. doi.org/10.1111/j.1472-8206.2004.00234.x.

Williamson, A., Hoggart, B., 2005. Pain: a review of three commonly used pain rating scales. J. Clin. Nurs. 1994, 798-804. 